\title{
A Comparative Study of Three Marker Detection Algorithms in Electron Tomography
}

\author{
Patrick Trampert ${ }^{1,2}$, Sviatoslav Bogachev ${ }^{1,2}$, Tim Dahmen ${ }^{1,2}$ and Philipp Slusallek ${ }^{1,2}$ \\ 1. German Research Center for Artificial Intelligence GmbH (DFKI), 66123 Saarbrücken, Germany. \\ 2. Saarland University, 66123 Saarbrücken, Germany.
}

We compared three commonly used algorithms for detecting fiducial markers in electron microscopy images [1]. The algorithms were implemented in a unified codebase in the software package Ettention [2] to ensure comparability of results without the influence of software. Several evaluation metrics were introduced to assess the capabilities of the algorithms on basis of four datasets. We showed, that depending on a dataset, different algorithms performed best. This proved, that the choice of a marker detection algorithm highly depends on the properties of a dataset to be analyzed, which makes it difficult to achieve best possible marker detection capabilities on a wide range of datasets with varying properties. Hence, more sophisticated marker detection methods may be needed to ensure the proper working of subsequent steps like alignment.

Marker detection capabilities of two cross-correlation based algorithms with template matching [3] and with pattern averaging [4], both also with additional filtering of false positives, as well as one convolution-based algorithm [5] were compared based on four datasets with discriminating properties and varying complex environment for expressive testing capabilities. The datasets covered a wide range of resolutions for both image resolution and marker resolution to ensure robustness and scalability. We compared the algorithms regarding their ability to find the right centre coordinate of markers as well as the reliability that detected markers are real markers in terms of sensitivity and in terms of making few mistakes. For unification of the number of identified markers and filtering of noise, all marker candidates were ranked by each algorithms individual score and the top 5\% were chosen as identified markers.

Experiments showed that dataset and application determine the algorithm to choose. Cross-correlation based methods find most real markers, but also introduce many falsely detected markers, which can be partly overcome with additional filtering that also filters out some real markers. The approach with pattern averaging dominates regarding the detection of true coordinates of markers. The convolutionbased method is very good at finding only real markers without introducing many falsely detected markers, however, it also misses many real markers.

The results of our study showed, that marker detection is also an important step to be considered and evaluated independently of further proceedings, which has not been done in any of the evaluated approaches. Depending on the dataset, results of complete pipelines that include marker detection, e.g. alignment, may heavily be influenced by the choice of the marker detection algorithm. The study showed that a higher awareness of that fact could lead to an improvement of many applications which rely on marker detection steps. Further research towards better marker detection algorithms that are able to adapt to a given dataset or majority frameworks of different marker detection algorithms could support such improvements. [7] 


\section{References:}

[1] P Trampert et al, Microsc. Microanalysis, 2015 Dec; 21(6): 1591-1601

[2] T Dahmen et al, Ultramicroscopy, 2016 Feb, vol. 161: 110-118.

[3] Brandt et al, J. Struct. Biol., 2001 Jan, vol. 133: 10-22.

[4] Amat et al, J. Struct. Biol., 2008: vol. 161: 260-275.

[5] Cao et al, J. Electron Microsc., 2011 Jan, vol. 60: 39-46.

[6] Diebolder et al, 2014 Mar, vol. 343: 1260-3.

[7] Research has been funded within the European Research Project NOTOX (FP7-267038). The authors thank the DFKI GmbH for additional funding and for providing the necessary infrastructure.
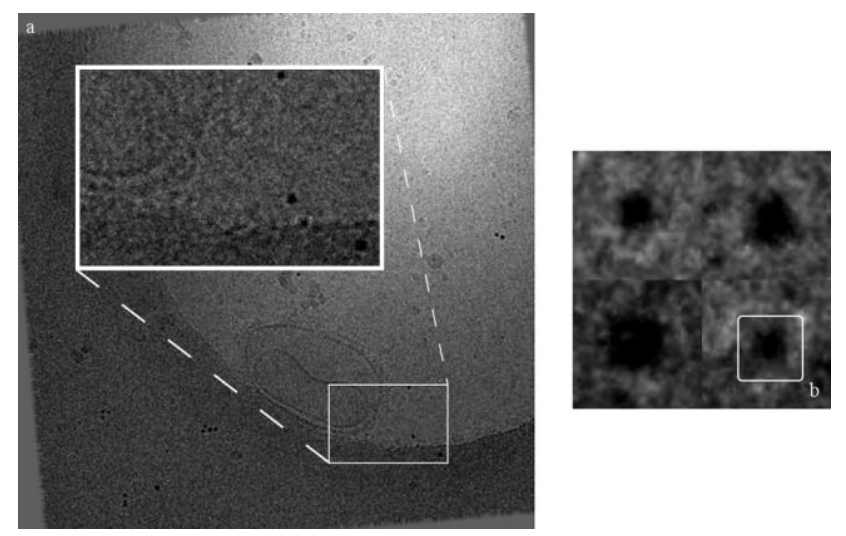

Figure 1. EMPIAR-10009, 120604_C4dep_10 [6], a) complete slice 11 and region of interest, b) selected marker candidates. The lower right candidate is an example of a structure that looks like a marker but it is no marker.
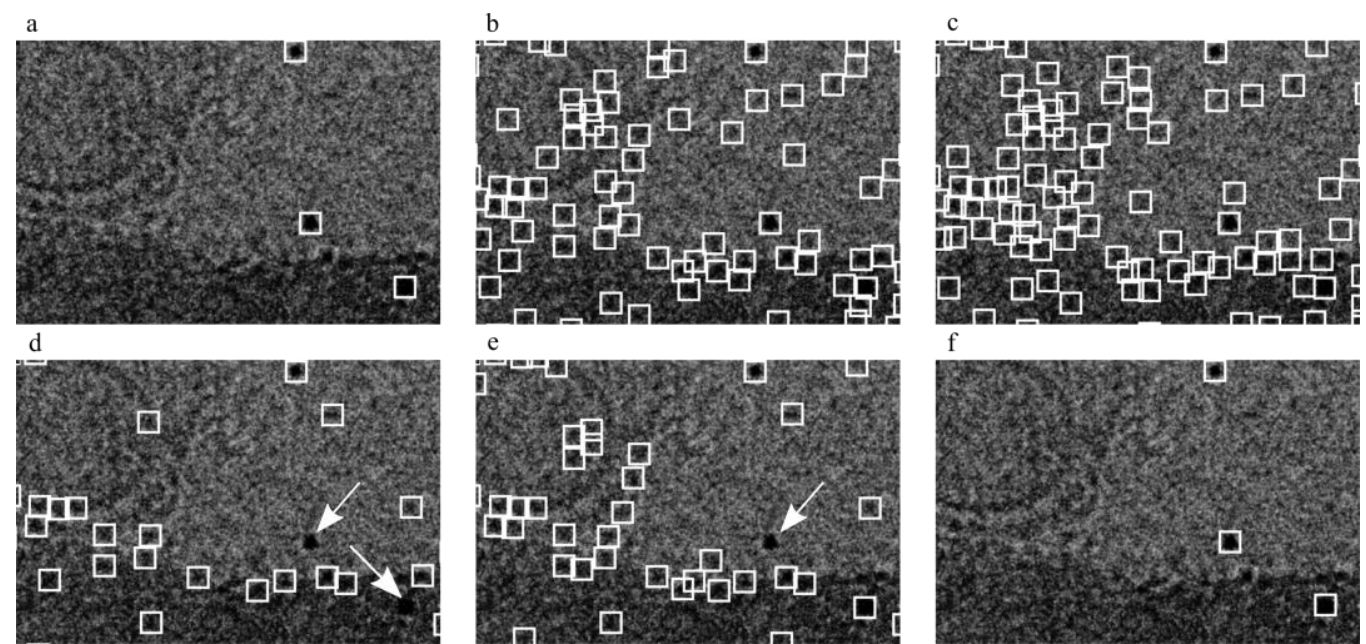

Figure 2. Detected markers of EMPIAR dataset extract of slice 11. a) manual detection as ground truth, b) method from [3], c) method from [4], d) method from [3] with additional filtering, e) method from [4] with additional filtering, f) method from [5]. The arrows in d) and e) are examples for removed true markers through filtering. 